Supplemental Material

for

\title{
Structural studies of Trypanosoma brucei RNA-editing ligases and their binding partner proteins
}

\author{
Alireza Shaneh ${ }^{1,4}$, Enrico O. Purisima ${ }^{2,3,4}$, Reza Salavati $^{1,3,4}$ and Traian Sulea ${ }^{1,2, *}$ \\ ${ }^{1}$ Institute of Parasitology, McGill University, 21111 Lakeshore Road, Ste. Anne de \\ Bellevue, H9X 3V9 Quebec, Canada \\ ${ }^{2}$ Human Health Therapeutics, National Research Council Canada, 6100 Royalmount \\ Avenue, Montreal, H4P 2R2 Quebec, Canada \\ ${ }^{3}$ Department of Biochemistry, McGill University, McIntyre Medical Building, 3655 \\ Promenade Sir William Osler, Montreal, H3G1Y6 Quebec, Canada \\ ${ }^{4}$ McGill Centre for Bioinformatics, McGill University, Bellini Building, 3649 Promenade \\ Sir William Osler, Montreal, H3GOB1 Quebec, Canada \\ * Corresponding author: traian.sulea@ cnrc-nrc.gc.ca, (514) 496-1924.
}




\section{Supplemental text}

Additional protein-RNA contacts including those distant from the ligation active site

In L1, the guanidinium group of Arg68 interact with the backbone atoms of $\mathrm{gC}(+7)$ and $\mathrm{gC}(+8)$ (Fig. S2). Furthermore, T91 interacts with the backbone group of the terminal and penultimate nucleotides (Fig. S3). The interaction between Asn92 and the backbone atom of the terminal nucleotide is shown in Figure S4. The amino group of Lys110 interacts with the nucleotide next to penultimate nucleotide (Fig. S5). The amine group of Gln193 makes the hydrogen bonds with the oxygen of sugar moiety of the terminal residue (Fig. S6). The interactions of Asn301 and Ser303 with the sugar moiety and backbone of $\mathrm{gC}(+7)$, respectively, are shown in Figure S7. Lys307 interacts with the oxygen atoms of $\alpha$-phosphorus in the nicked nucleotide, while Arg309 interacts with the backbone of the nucleotide next to the adenylated base (Fig. S8).

In L2, Arg27 contacts with the backbone of $\mathrm{gU}(+4)$ (Fig. S9). In addition, Lys78 interacts with the backbone of $\mathrm{C}(-3)$ (Fig. S10), and Ser80 interacts with the backbone of $\mathrm{C}(-3)$. Furthermore, Thr158 interacts with the backbone atoms of $\mathrm{gG}(-2)$ and $\mathrm{gG}(-3)$ (Fig. S11). Lys271 interacts with bridging oxygen between the nicked nucleotide and one nucleotide after the adenylated nucleotide (Fig. S12). 


\section{Supplemental figures}

Fig. S1. Molecular dynamics time series for distances between (A) $\mathrm{Mg}^{2+}$ ion and 3'-OH oxygen atom to be ligated, (B) $\mathrm{Mg}^{2+}$ ion and the oxygen atom from the 5'-PO4 group to be ligated, and (C) 3'-OH oxygen atom and 5'-PO4 phosphorus atom from the two RNA ends to be ligated, and (D) the angle for nucleophilic attack of 3'-OH oxygen atom to the 5'- $\mathrm{PO}_{4}$ phosphorus atom with the displacement of the oxygen atom of the AMP leaving group. Data from three MD-replicates are plotted for $\mathrm{L} 1^{\mathrm{NT}}$-dsRNA (black lines) and L2 ${ }^{\mathrm{NT}}$-dsRNA (red lines) complexes. See Table 1 in the main paper for average and standard deviation values.

Fig. S2. Interaction of $\mathrm{Arg} 68$ with $\mathrm{gC}(+7)$ and $\mathrm{gC}(+8)$ in L1-RNA complex.

Fig. S3. Interaction of Thr91 with terminal and penultimate nucleotides (L1-RNA complex).

Fig. S4. Interaction of Asn92 with terminal nucleotide (L1-RNAa complex).

Fig. S5. Interaction of Lys110 with C(-3) in L1-RNA complex.

Fig. S6. Interaction of Gln193 with terminal nucleotide (L1-RNA complex).

Fig. S7. Interaction of Asn301 and Ser303 with gC(+7) (L1-RNA complex).

Fig. S8. Interaction of Lys307 and Arg309 with the nicked nucleotide and A(+1) (L1-RNA complex).

Fig. S9. Interaction of $\mathrm{Arg} 27$ with $\mathrm{gU}(+4)$ in L2-RNA complex.

Fig. S10. Interaction of Lys78 with C(-3) (L2-RNA complex).

Fig. S11. Interaction of Thr158 with $\mathrm{gG}(-2)$ and $\mathrm{gG}(-3)$ (L2-RNA complex).

Fig. S12. Interaction of Lys271 with the nicked nucleotide and A(+1) (L2-RNA complex).

Fig. S13. Overall geometry of the simulated ligase-RNA complexes. Superposition of T4-Rnl2DNA-RNA crystal structure shown in green (Nandakumar et al. 2006) onto (A) L1dsRNA (cyan, this study), and (B) L2-dsRNA (magenta, this study).

Fig. S14. Superposition of L1-RNA complex (cyan) onto $C \rightarrow G$ variant model (green) and interaction of Gln193 with bases of cytosine and guanine. The distance between the amino group of Gln193 and the oxygen atom of each base is shown in dotted lines. The distance value is in Angstrom.

Fig. S15. Superposition of L2-RNA complex (cyan) onto $C \rightarrow G$ variant model (green) and interaction of Gln157 with bases of cytosine and guanine. The distance between the amino group of Gln157 and the oxygen atom of each base is shown in dotted line. The distance values are in Angstrom.

Fig. S16. Sequence alignment between the OB-folded domains of the KREPA1 and KREPA2 homologous proteins. Secondary structure elements correspond to the modeled structure of the $\mathrm{A} 1^{\mathrm{OB}}$ domain bound to the $\mathrm{L} 2^{\mathrm{NT}}$-dsRNA complex. Highlighted residues are 
predicted to establish direct contacts with dsRNA. Note that the insertion present in $\mathrm{A} 1^{\mathrm{OB}}$ relative to $\mathrm{A} 2^{\mathrm{OB}}$ and corresponding to the long $\mathrm{L} 23$ loop is not included in the present model, which follows the construct used in the published crystal structure of $\mathrm{A} 1^{\mathrm{OB}}$ (PDB code 4DK6). 
A

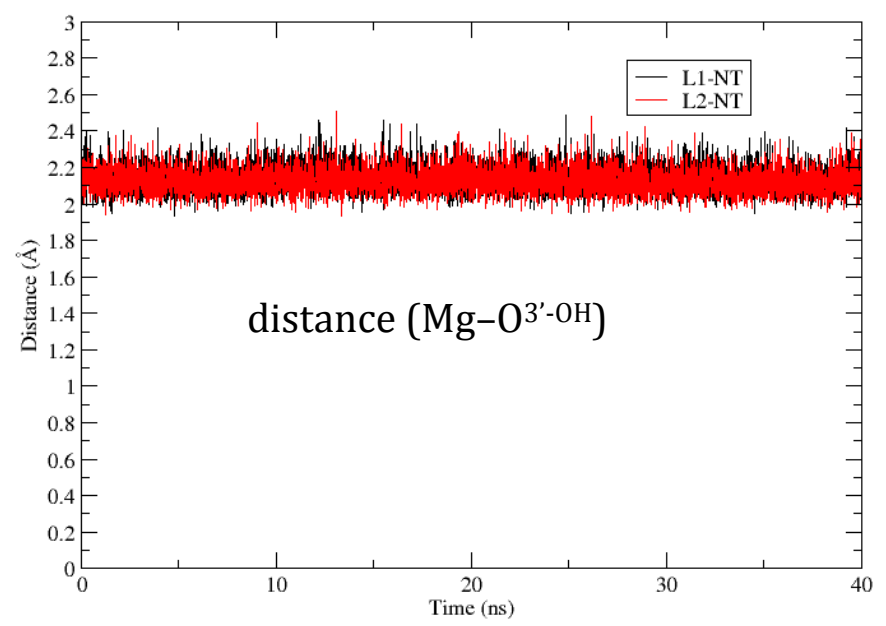

C

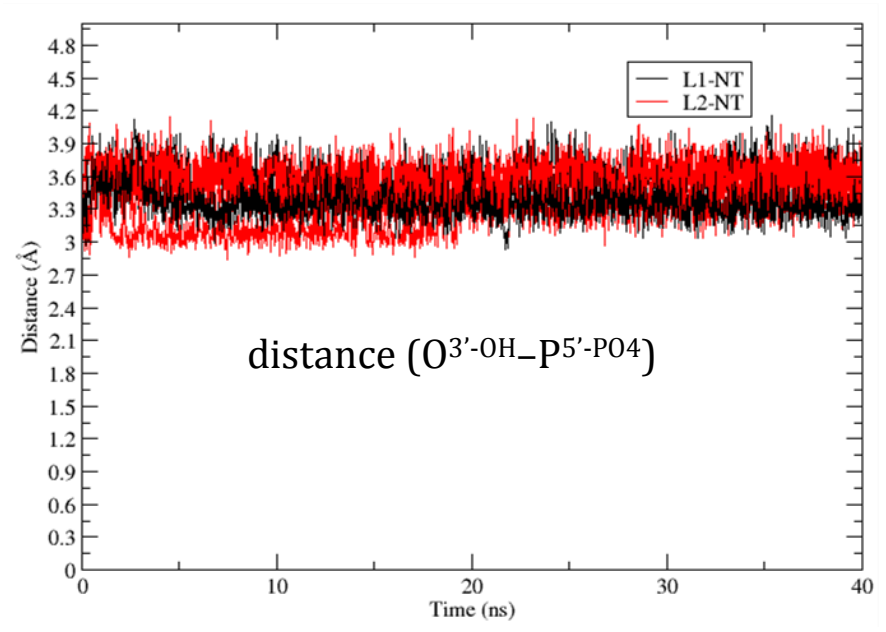

B

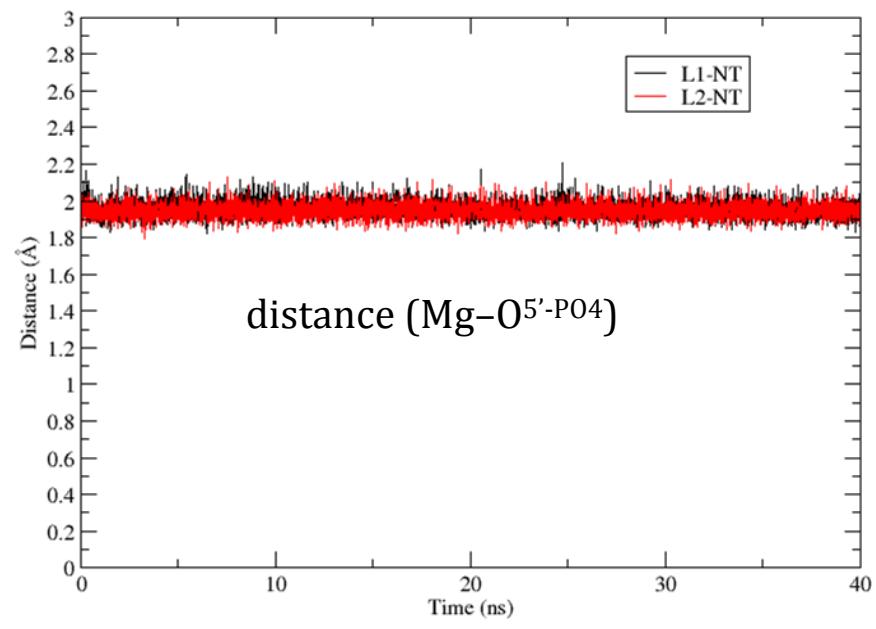

D

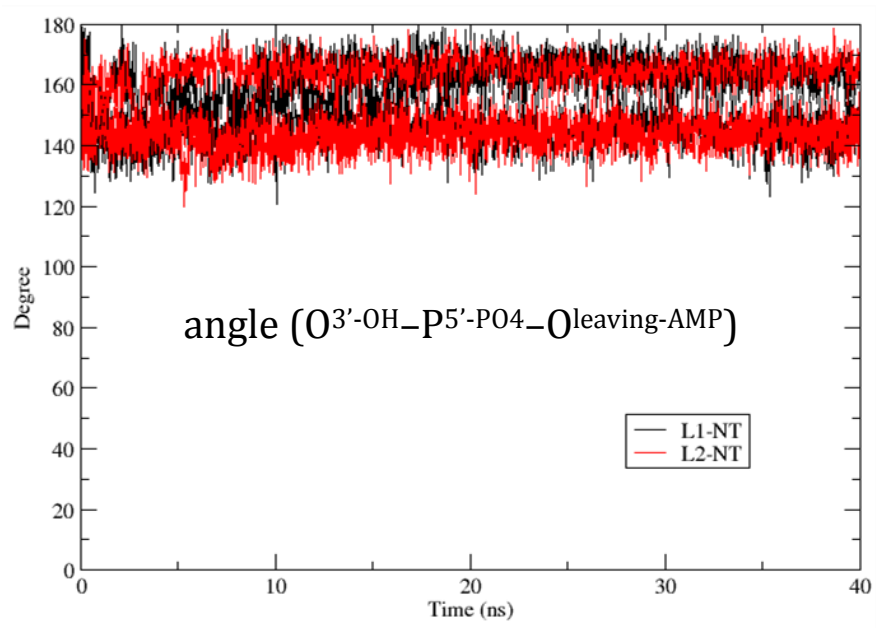

Fig. S1 


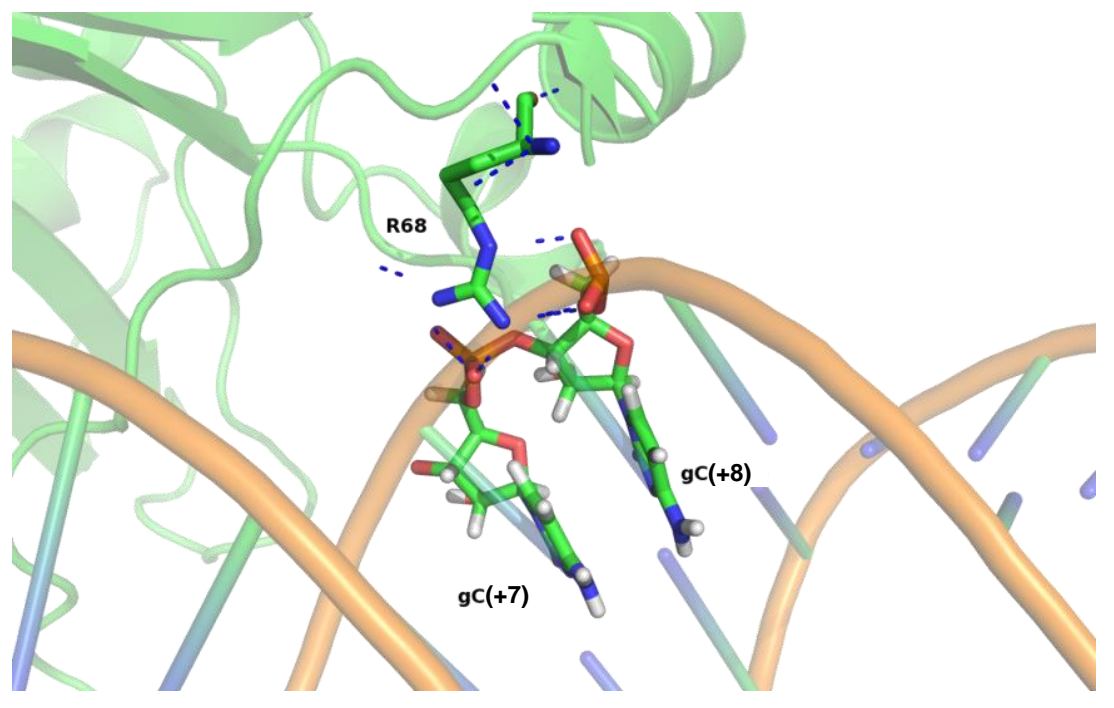

Fig. S2

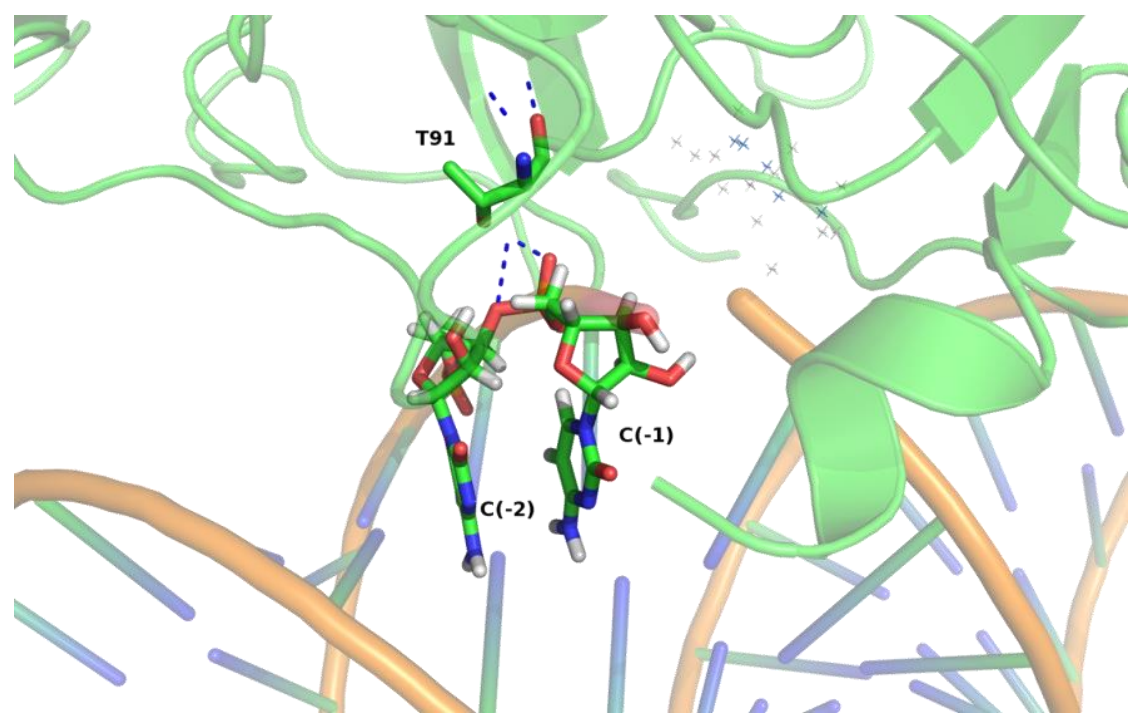

Fig. S3 


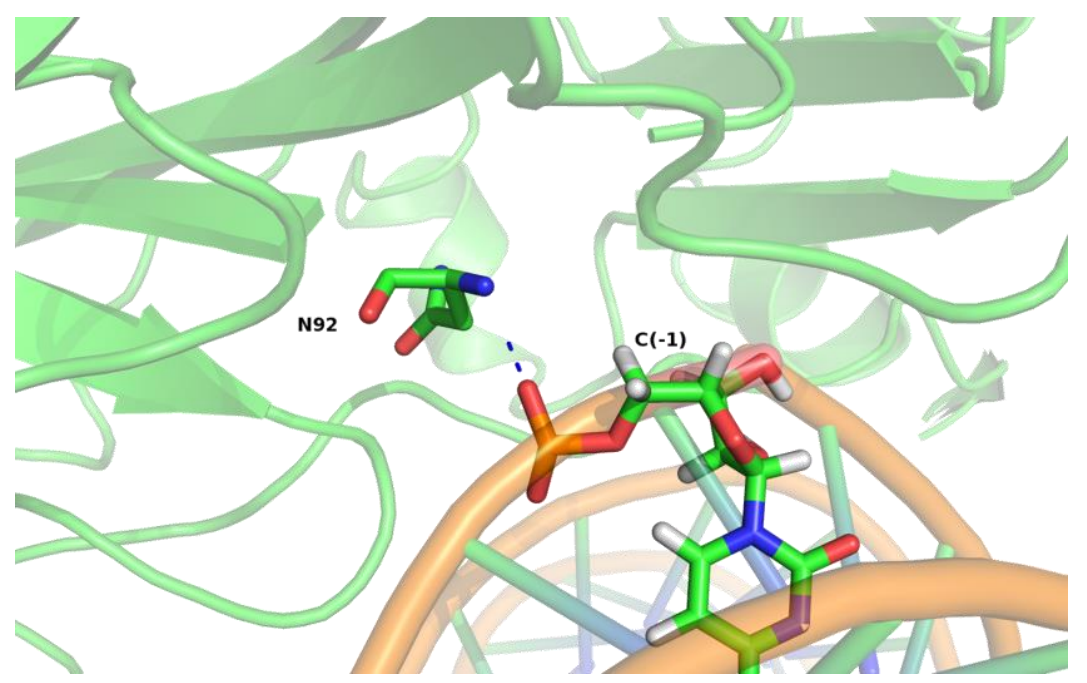

Fig. S4

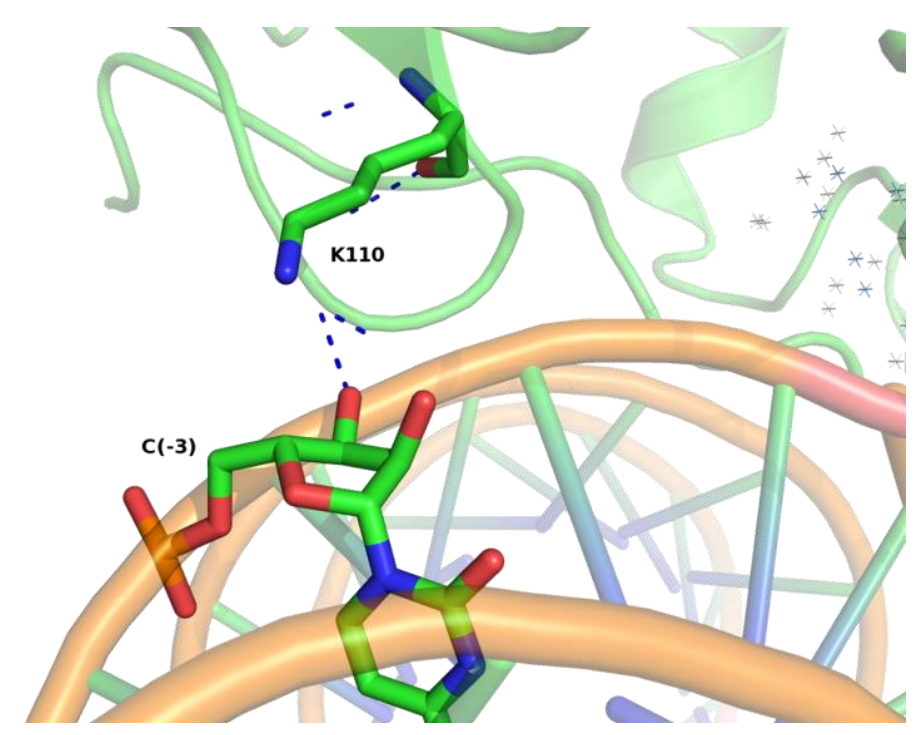

Fig. S5 


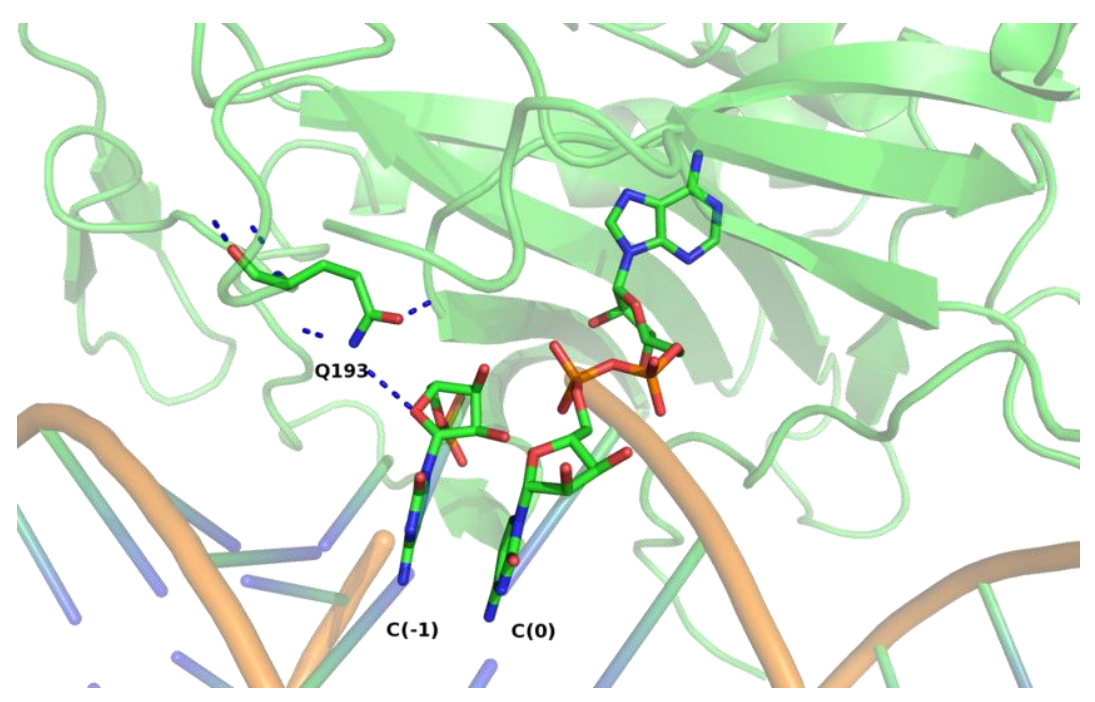

Fig. S6

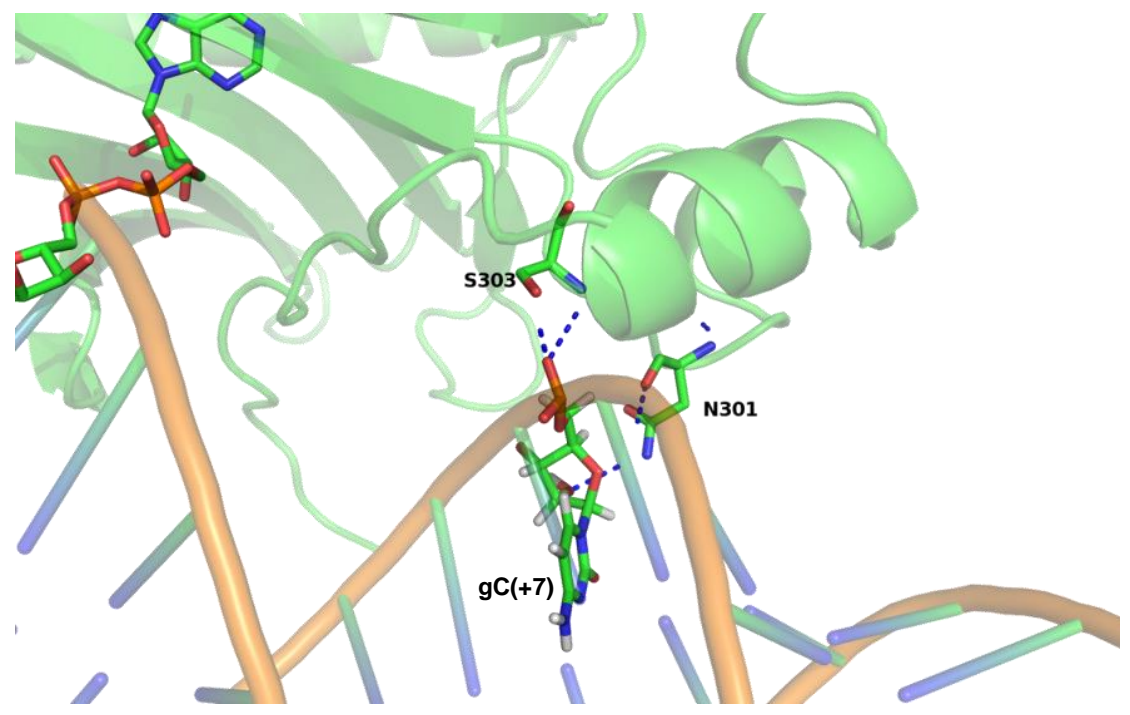

Fig. S7 


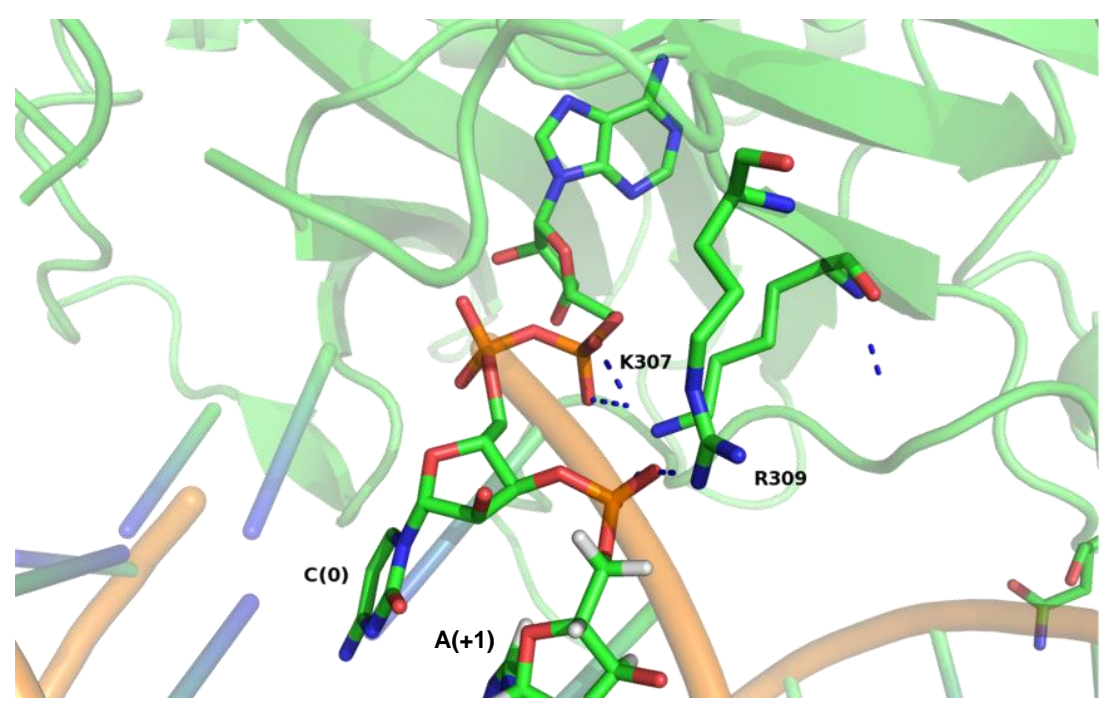

Fig. S8

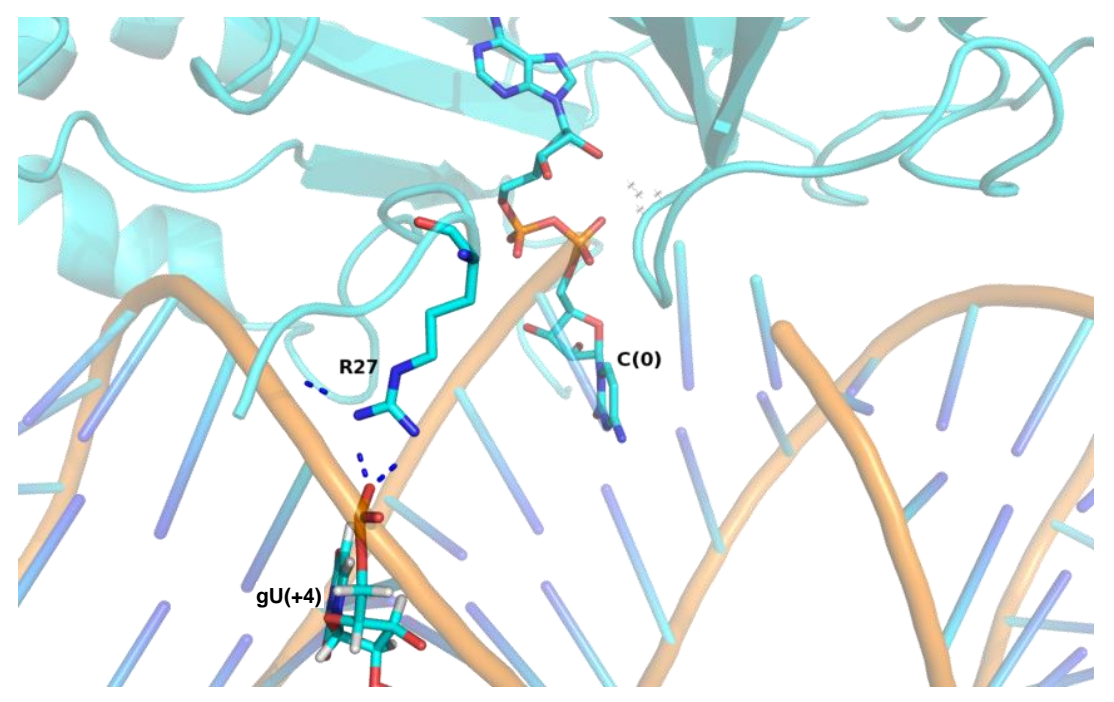

Fig. S9 


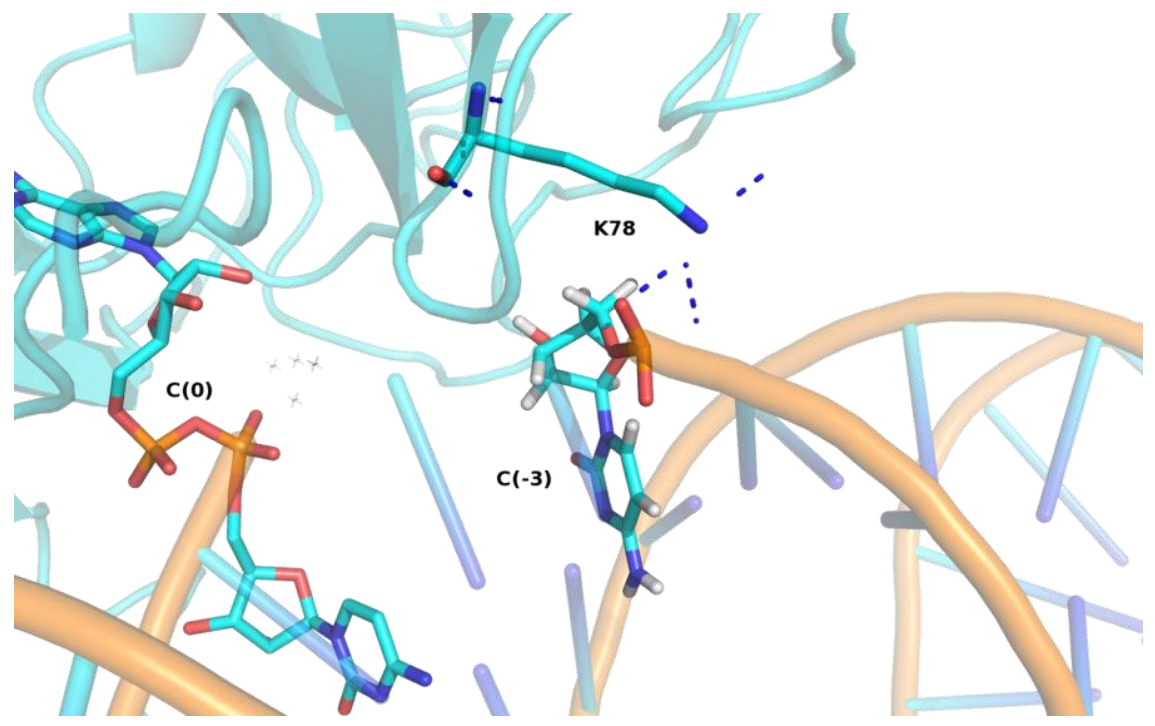

Fig. S10

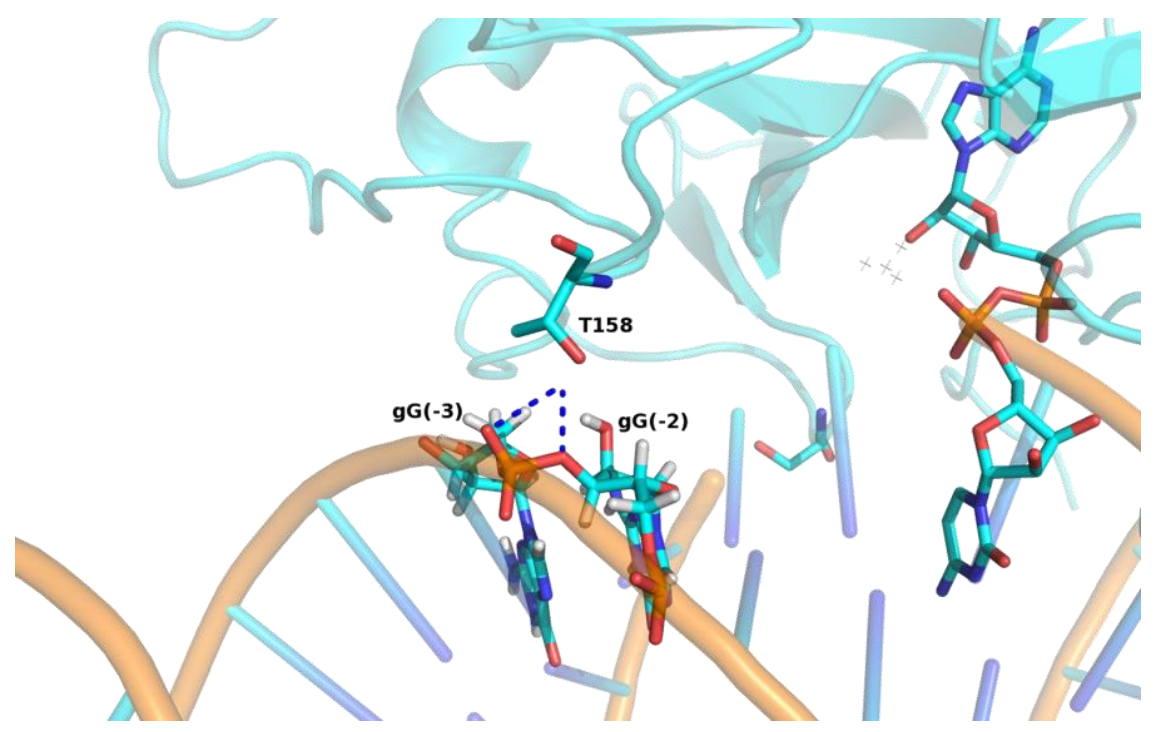

Fig. S11 


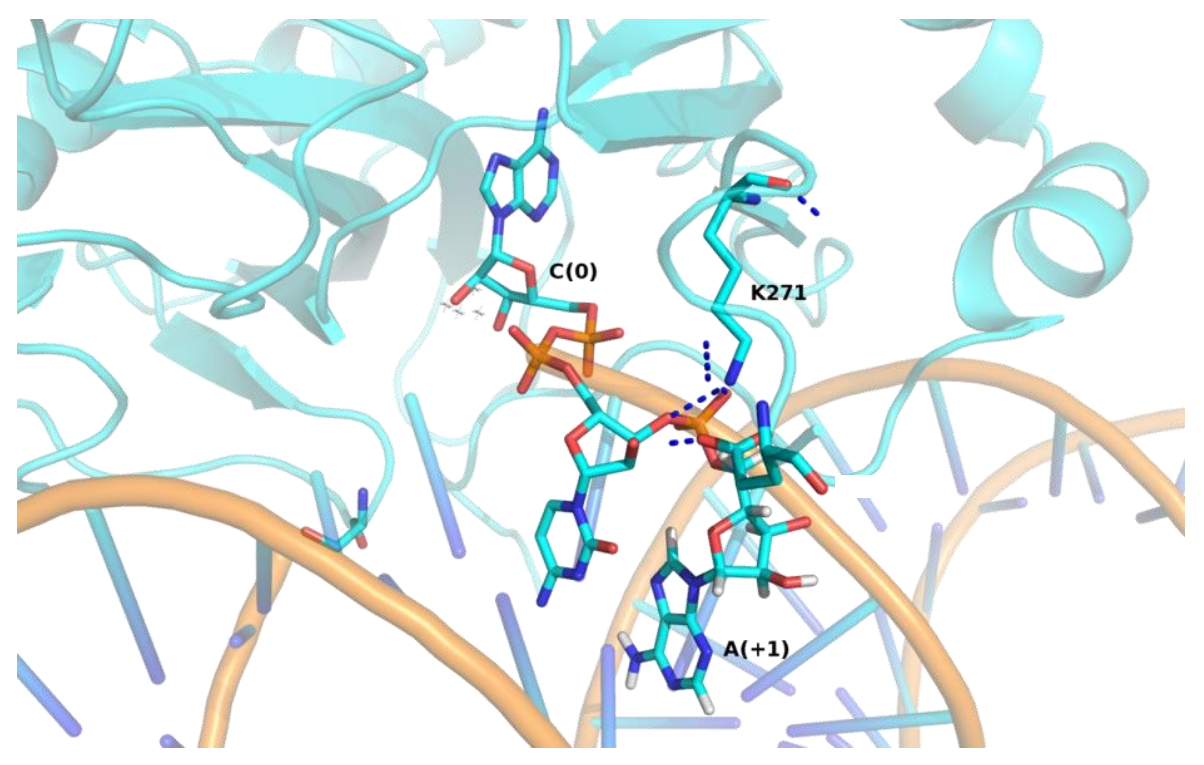

Fig. S12 
A

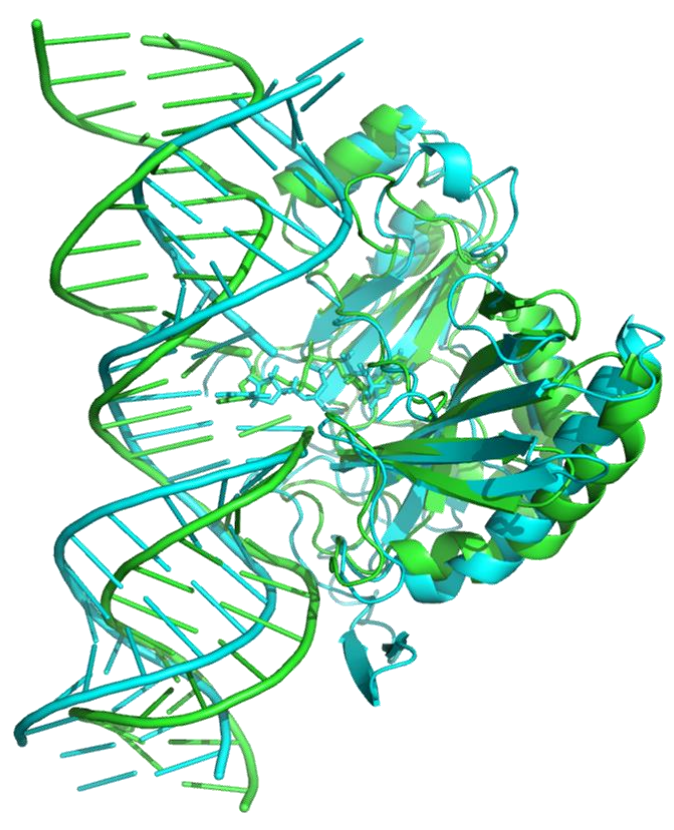

B

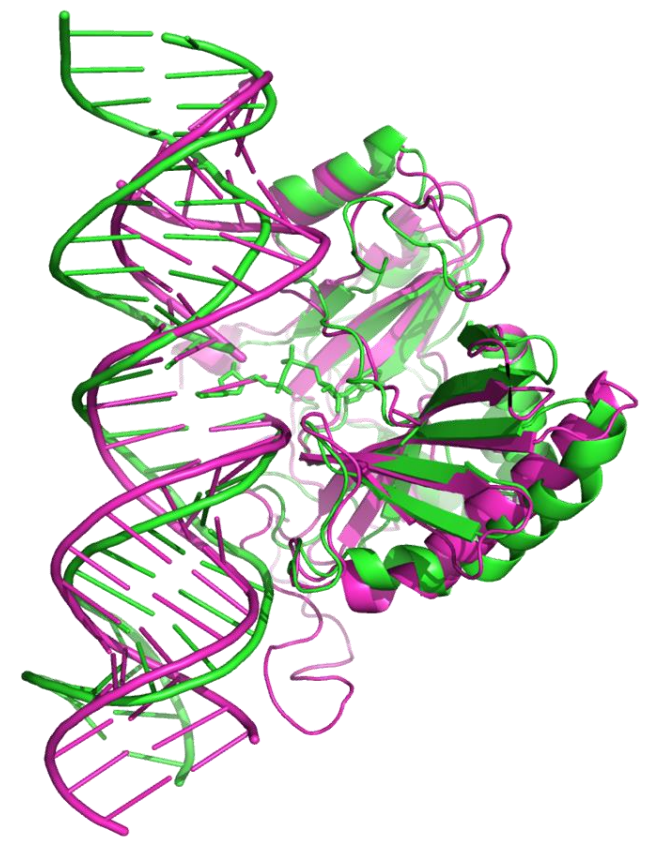

Fig. S13 


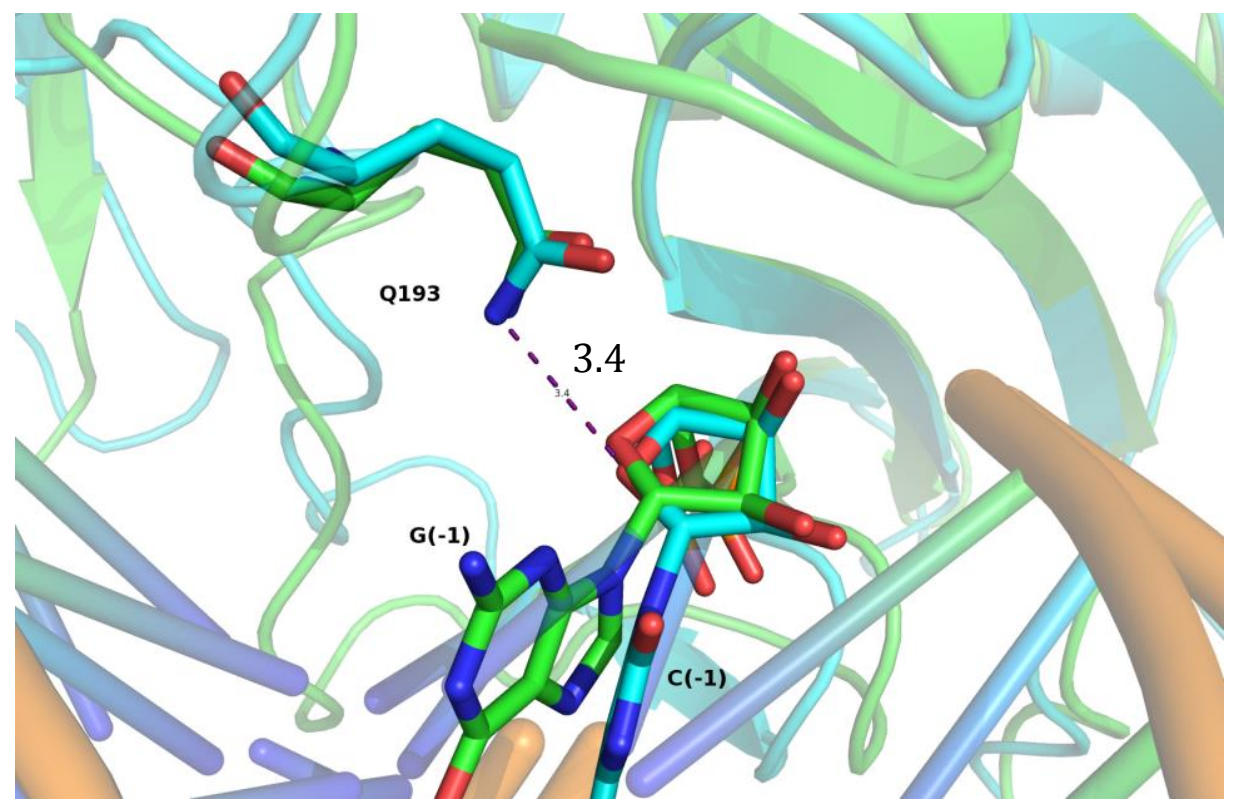

Fig. S14

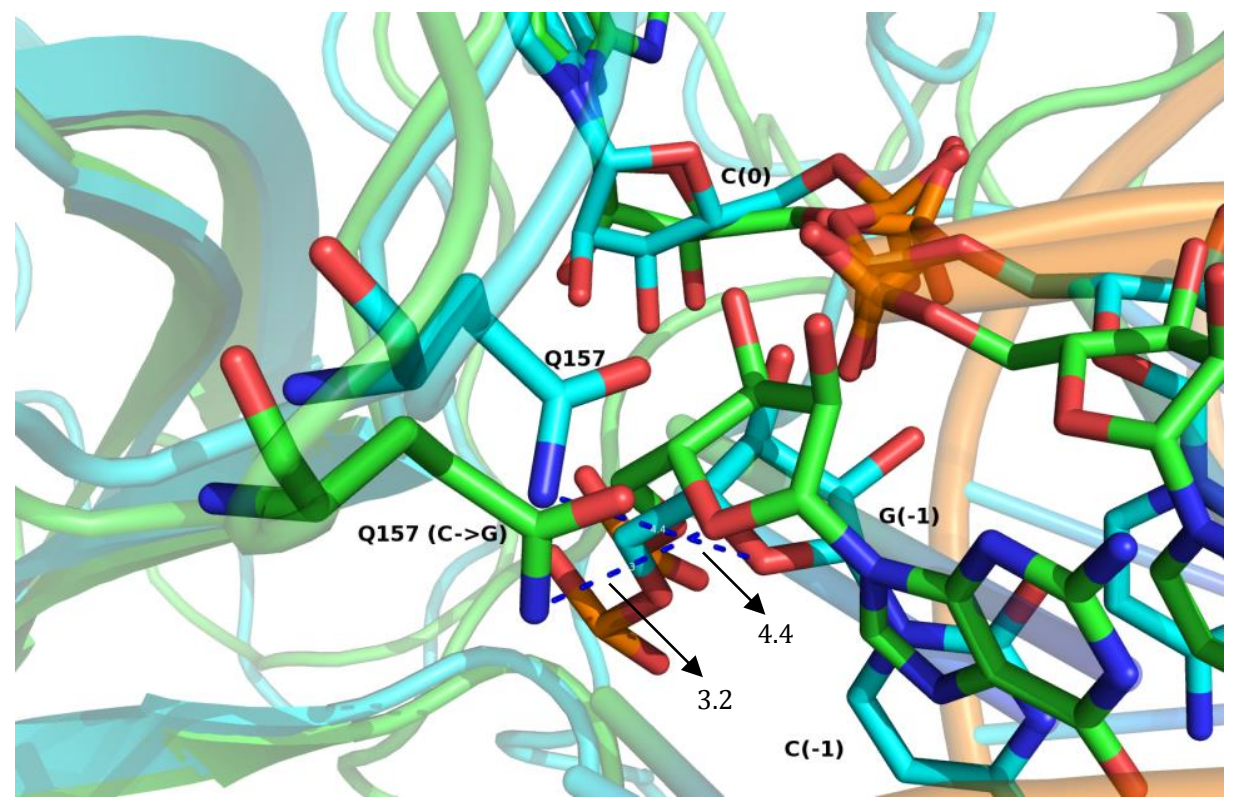

Fig. S15 


\begin{abstract}
Model-sS <---- $\beta 1---->\quad$ L12 <- $32-->\quad$ L23
A1-OB SNALMIGRIADVQHGFLGAMTVTQYVLEVDGDERINSKGVTTPASACTPDPASTKAVEAKGEEGEVVE

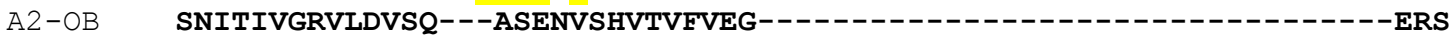

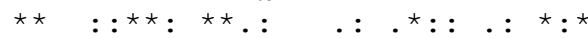

Model-sS $\quad<-\beta 3->\quad<-\alpha 1-->\quad<--\beta 4--->\quad$ L45 $<\beta 5>$ L56 < <6>

A1-OB PEKEFIVIRCMGDNFPASLLKDQVKLGSRVLVQGTLRMNRHVDDVSKRLHAYPFIQVVPPLGYVKVV

A2-OB GEEETLTLCCFGE--VSQKIRGTLKRNATIFASGTLRLHPVYEASNNKYYVSPVVHVSMPTGTLAVI

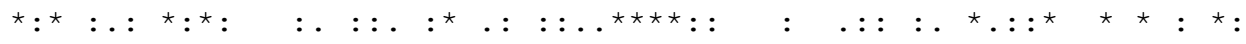

Fig. S16 


\section{Supplemental table}

Table S1. The mean \pm standard deviation of calculated binding free energies (SIE values, in $\mathrm{kcal} / \mathrm{mol}$ ) based on three MD-trajectory replicates (data computed over the last $20 \mathrm{~ns}$ of each trajectory).

\begin{tabular}{ccccc}
\hline & Replicate1 & Replicate2 & Replicate3 & Average \\
\hline$M g^{2+}$ part of ligase & & & & \\
L1 $^{\mathrm{NT}}$-dsRNA & $-14.74 \pm 1.25$ & $-19.17 \pm 1.25$ & $-18.71 \pm 1.41$ & $-17.54 \pm 2.48$ \\
L2 $^{\mathrm{NT}}$-dsRNA & $-19.10 \pm 1.57$ & $-20.21 \pm 1.54$ & $-21.14 \pm 1.50$ & $-20.15 \pm 1.02$ \\
\hline$M^{2+}$ part of $R N A$ & & & & \\
L1 $^{\mathrm{NT}}$-dsRNA & $-12.67 \pm 1.61$ & $-16.67 \pm 1.20$ & $-15.96 \pm 1.41$ & $-15.10 \pm 2.13$ \\
L2 $^{\text {NT }}$-dsRNA & $-16.48 \pm 1.55$ & $-17.15 \pm 1.48$ & $-18.15 \pm 1.37$ & $-17.26 \pm 2.41$ \\
\hline
\end{tabular}

